\title{
Perturbative moduli stabilisation in type IIB/F-theory framework
}

\author{
Ignatios Antoniadis $^{1,2, a}$, Yifan Chen ${ }^{1, b}$, George K. Leontaris ${ }^{3, c}$ \\ ${ }^{1}$ Laboratoire de Physique Théorique et Hautes Énergies, LPTHE, Sorbonne Université, CNRS, 4 Place Jussieu, 75005 Paris, France \\ ${ }^{2}$ Albert Einstein Center, Institute for Theoretical Physics, University of Bern, Sidlerstrasse 5, 3012 Bern, Switzerland \\ ${ }^{3}$ Physics Department, University of Ioannina, 45110 Ioannina, Greece
}

Received: 5 April 2018 / Accepted: 15 September 2018 / Published online: 24 September 2018

(C) The Author(s) 2018

\begin{abstract}
We propose a new mechanism of (geometric) moduli stabilisation in type IIB/F-theory four-dimensional compactifications on Calabi-Yau manifolds, in the presence of 7-branes, that does not rely on non-perturbative effects. Complex structure moduli and the axion-dilaton system are stabilised in the standard way, without breaking supersymmetry, using 3-form internal fluxes. Kähler class moduli stabilisation utilises perturbative string loop corrections, together with internal magnetic fields along the D7branes world-volume leading to Fayet-Iliopoulos D-terms in the effective supergravity action. The main ingredient that makes the stabilisation possible at a de Sitter vacuum is the logarithmic dependence of the string loop corrections in the large two-dimensional transverse volume limit of the 7-branes.
\end{abstract}

\section{Introduction}

The String Theory landscape comprises an enormous number of vacua, however, not all of them are consistent with the cosmological data and the relevant for particle physics effective $N=1$ supergravity theories. Many of them are characterised by anti-de-Sitter (AdS) minima, predicting a negative cosmological constant, in contradiction with the existing evidence of the accelerated expansion of the universe. In order to obtain a consistent supersymmetric vacuum we must seek string compactifications with a de Sitter (dS) minimum and stabilise the various moduli fields which are ubiquitous in string compactifications.

In type IIB string theory in particular, compactified on a Calabi-Yau threefold, the complex structure moduli and the axion-dilaton/ten-dimensional (complexified) string coupling appear in the superpotential induced when 3-form

\footnotetext{
a e-mail: antoniad@lpthe.jussieu.fr

b e-mail: yifan.chen@1pthe.jussieu.fr

ce-mail: leonta@uoi.gr
}

fluxes are turned on and can be fixed in a supersymmetric way [1,2]. ${ }^{1}$ The Kähler class moduli on the other hand, such as the Calabi-Yau volume, remain undetermined because being $(1,1)$ forms - they do not appear in the flux induced superpotential. The resulting effective supergravity has constant superpotential and, thus, a non-vanishing gravitino mass term and vanishing scalar potential, due to the no-scale structure of the tree-level Kähler potential of the Kähler class moduli that remain massless and undetermined. Their stabilisation requires their appearance in the superpotential and, a usual way to realise it, is to include non-perturbative corrections [5]. These are in general model dependent related, for instance, to gaugino condensation of the gauge group of $D 7$-branes [6,7]. In the simplest case, to realise a sufficiently large volume in a well controlled regime, a fine tuning of the coefficients in the resulting superpotential generated by the fluxes is required.

Moreover, higher order $\alpha^{\prime}$ corrections are taken into account and break the no-scale structure of the Kähler potential [8]. One-loop corrections to the Kähler potential may also be included [9-17]. In most cases, dS vacua can only be obtained by 'uplifting' the vacuum energy in the presence of anti- $D 3$ branes ( $\overline{D 3}$-branes for short), which break though supersymmetry explicitly (KKLT scenario [5]). This situation can, in principle, be remedied if instead of $\overline{D 3}$ branes, D-term contributions are taken into account in the effective action [18-24], emerging from internal magnetic fluxes along the $D 7$-branes world volume. String realisations improving the KKLT scenario are also possible within the so-called large volume scenario in Calabi-Yau compactifications [25,26].

In the present work we take a different path and, working in the framework of type IIB/F-theory, we consider possible contributions to the Kähler potential due to the presence of space-time filling $D 7$-branes. Recall that 7-

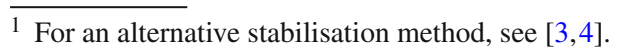


branes are fundamental objects in F-theory and certain configurations of them determine the gauge symmetry of the effective theory. Moreover, an important class of matter fields resides on Riemann surfaces which can be interpreted as the intersection locus of $D 7$-branes. In such configurations, anomalous $U(1)$ symmetries, associated with intersecting branes, are frequently present and the resulting chiral spectrum of the four-dimensional theory usually induces Fayet-Iliopoulos (FI) D-terms to the effective potential. The importance of introducing D-term contributions is that these are always positive and can in principle uplift the potential, generating a $\mathrm{dS}$ minimum with all moduli fixed.

It turns out though that D-terms are not sufficient to stabilise all moduli, at least when charged fields on D7branes have vanishing expectation values. The basic additional ingredient that comes in rescue is quantum corrections to the Kähler potential stemming from the presence of the D7-branes. For large transverse volume, as is the case of such configurations, these corrections become crucial and cannot be neglected. They display a logarithmic dependence on the modulus associated with the transverse dimension [27]. The logarithmic dependence is quite general in the presence of two large transverse dimensions, as shown for example in [28]. Combining these effects with D-term contributions, we show that all Kähler moduli can be stabilised in a dS vacuum of broken supersymmetry.

The layout of this paper is as follows. In Sect. 2, we start with a general overview of the moduli stabilisation problem, we introduce the effective supergravity and present the leading quantum corrections, perturbative in $\alpha^{\prime}$ and in the string coupling (Sect. 2.1). We discuss in particular their dependence on the transverse volume of the $D 7$-branes that grows logarithmically at large distances. We then introduce the Dterm contributions to the effective potential (Sect. 2.2). In Sect. 3, we work out the minimisation conditions and the Kähler moduli stabilisation. We first present the simple example of a single $D 7$-brane which brings two moduli that can be chosen to be the total volume of the Calabi-Yau manifold and the volume transverse to the brane (Sect. 3.1). We show how the latter can be stabilised using the logarithmic loop corrections, but not the former. Full stabilisation in a dS minimum can be achieved only in the general case of three intersecting $D 7$-branes with corresponding Dterms. Indeed, the total volume can be stabilised by the logarithmic corrections (Sect. 3.2), while all moduli are fixed when D-terms are included, as shown in Sect. 3.3. Finally, Sect. 4 contains our conclusions, while in the Appendix we show why in the case of one D7-brane, one cannot find a dS minimum in the whole parameter space of the model.

\section{General overview}

In IIB string theory, appropriate 3-form fluxes generate a superpotential given by [29]:

$\mathcal{W}=\int G_{3} \wedge \Omega$.

In the above, the $G_{3}$ flux is defined as $G_{3}=F_{3}-S H_{3}$, where $F_{3}, H_{3}$ are the Ramond-Ramond (RR) and NeveuSchwarz (NS) 3-form fluxes, $S=C_{0}+i e^{-\phi} \equiv C_{0}+i / g_{s}$ is the axion-dilaton field associated with the ten-dimensional string coupling $g_{s}$, and $\Omega$ is the holomorphic Calabi-Yau $(3,0)$-form dependent on complex structure moduli $z_{a}$ [30]. Clearly, the superpotential (1) depends on $z_{a}$ and the axiondilaton $S$ but it is independent of the Kähler class moduli, as described for example in [31]. The conditions $D_{a} \mathcal{W}=0$, (where $D_{a}$ is the Kähler covariant derivative and the index $a$ runs over all moduli fields) fix all the complex structure moduli and the axion-dilaton. A generalization of Eq. (1) in the F-theory framework is straightforward $[32,33]$.

A wide class of solutions towards the stabilisation of Kähler class moduli rely on non-perturbative effects. This ingredient allows the appearance of the Kähler moduli in the superpotential and as a result, a potential is generated whilst their masses are determined from the minimisation procedure of the effective potential. The $\alpha^{\prime}$-corrections which generate $\mathcal{O}\left(\alpha^{\prime 3}\right)$ contributions to the Kähler potential, are also widely used. In the present analysis, we will take a different path and investigate the effects of string loop corrections to the Kähler potential which displays a dependence on the transverse volume of $D 7$-branes, as well as the D-terms potential depending on the world-volume of $D 7$-branes.

To set the stage, we start with the Kähler potential. Ignoring for the moment $\alpha^{\prime}$ or string loop corrections, it can be written as a simple separable form

$\mathcal{K}_{0}=\mathcal{K}_{0}\left(T_{i}\right)+\mathcal{K}\left(S, z_{a}\right)$,

where $T_{i}$ are the Kähler moduli, $S$ the axion-dilaton $S=$ $C_{0}+i e^{-\phi}$, and $z_{a}$ the complex structure moduli respectively. The two components of $\mathcal{K}$ are

$$
\begin{aligned}
\mathcal{K}_{0}\left(T_{i}\right) & =-\sum_{i=1}^{3} \ln \left(-i\left(T_{i}-\bar{T}_{i}\right)\right) \\
\mathcal{K}\left(S, z_{a}\right) & =-\ln (-i(S-\bar{S}))-\ln \left(i \int \Omega \wedge \bar{\Omega}\right) .
\end{aligned}
$$

As can be readily seen, Eq. (3) satisfies the no-scale condition

$$
\sum_{I, J=T_{i}} \mathcal{K}_{0}^{I \bar{J}} \partial_{I} \mathcal{W} \partial_{\bar{J}} \mathcal{W}=3
$$

and therefore the potential can be written as

$$
V=\sum_{I, J \neq T_{i}} e^{\mathcal{K}}\left(D_{I} \mathcal{W} \mathcal{K}_{I \bar{J}}^{-1} D_{\bar{J}} \mathcal{W}\right)
$$


with $D_{I} \mathcal{W}=\partial_{I} \mathcal{W}+\mathcal{W} \partial_{I} \mathcal{K}$. In the simplest scenario, the flux generated perturbative superpotential in Eq. (1) stabilises the complex structure moduli and the axion-dilaton field by using the supersymmetric conditions $D_{i} \mathcal{W}=0$. This leads to zero vacuum energy. Furthermore, because of the no scale structure of the Kähler potential, the Kähler moduli cannot be fixed from the supersymmetric minimisation of the superpotential. In order to stabilise the Kälher moduli, quantum corrections are usually included so that the no-scale invariance is violated and the total volume is fixed.

In addition, non-perturbative contributions originating from gaugino condensation or instanton effects, involve certain Kähler moduli in exponentially suppressed terms. Including such terms, the superpotential obtains the form

$\mathcal{W}=\mathcal{W}_{0}+\sum_{i=1}^{h_{+}^{1,1}} \Lambda_{i} e^{-\lambda_{i} T_{i}}$

The implementation of the conditions $D_{z_{a}} \mathcal{W}=0$ put all complex structure moduli and axion-dilaton at their minima, and so $\mathcal{W}_{0}, \Lambda_{i}$ and $\lambda_{i}$ are constants. The condition $D_{T_{i}} \mathcal{W}=0$ leads to a supersymmetric AdS minimum [5]. However, a drawback of this scenario is that the minimum is achieved only when the value of $\mathcal{W}_{0}$ is fine-tuned in order to balance the non-perturbative effects. In addition, as can be seen from (7), non-perturbative corrections are required for all 4-cylces involved, whilst the inclusion of $\overline{D 3}$ contributions to uplift the AdS minimum breaks supersymmetry explicitly.

A generalisation of the above scenario $[25,26]$ improving these deficiencies, is realised with an exponentially large volume, where in the simplest case of two Kähler moduli $\tau_{b}, \tau_{s}$ the Calabi-Yau volume takes the form $\mathcal{V}=\tau_{b}^{3 / 2}-\tau_{s}^{3 / 2}$. In the presence of non-perturbative corrections the Kähler potential and superpotential are given by

$$
\begin{aligned}
\mathcal{K}_{L V S} & =-2 \ln \left(\tau_{b}^{\frac{3}{2}}-\tau_{s}^{\frac{3}{2}}+\xi\right), \\
\mathcal{W}_{L V S} & =\mathcal{W}_{0}+\Lambda e^{-\lambda \tau_{s}} .
\end{aligned}
$$

The gaugino condensation and the $\alpha^{\prime}$ correction $\xi$ are necessary to stabilise both $\tau_{b}$ and $\tau_{s}$. However, as in the KKLT case, a mechanism is required to uplift it to a $\mathrm{dS}$ minimum.

In the next subsections, we will present an alternative scenario of Kähler moduli stabilisation which does not rely on (uncontrolable) non-perturbative corrections in the superpotential. The proposed mechanism is based on the observation that the effective action receives logarithmic corrections in the large (two dimensional) volume limit transverse to the D7-branes [27].
2.1 Effective supergravity, dualities and quantum corrections

We would like now to include the leading quantum corrections in the effective action presented above. These are perturbative in $\alpha^{\prime}$ or in the string coupling correcting the Kähler potential and the gauge kinetic functions. The former correspond to a constant shift of the internal volume proportional to the Euler number of the Calabi-Yau manifold, while the latter can be important only in the presence of large transverse volume of dimension less or equal than two, as in the configuration of $D 7$-branes that we consider in this work. In this case, one loop corrections to localised effective action terms in the open string channel grow logarithmically with the transverse volume [27]. Since such corrections have been computed explicitly in $N=1$ type I orientifolds [37], we will start by presenting them in this framework and then use T-dualities to derive the corresponding expressions in type IIB/F-theory context with intersecting $D 7$-branes.

Let us consider type I strings on a product of three 2-torii $\left(\prod_{i=1}^{3} T_{i}^{2}\right.$ ) with $D 9$-branes and in general three types of $D 5$ branes extended in the three non-compact spatial dimensions and along each one of the three $T_{i}^{2}$. The Kähler potential is

$\mathcal{K}=-\ln (S-\bar{S})-\sum_{i=1}^{3} \ln \left(T_{i}-\bar{T}_{i}\right)+\cdots$,

where the dots refer to contributions dependent on the complex structure that we omit in the following. The imaginary part of the various moduli are given by the inverse gauge couplings of the $D 9$ and $D 5_{i}$ branes, upon compactification in four dimensions:

$\operatorname{Im} S=\frac{1}{g_{9}^{2}}=e^{-\phi} v_{1} v_{2} v_{3} \quad \operatorname{Im} T_{i}=\frac{1}{g_{5 i}^{2}}=e^{-\phi} v_{i}$,

with $v_{i}$ the volume of $T_{i}^{2}$ in string units.

To go to the framework of type IIB/F-theory with three types of $D 7$-branes, one has to perform six T-dualities along all six internal directions. The $D 9$ then becomes $D 3$ while $D 5_{i}$ becomes $D 7_{i}$ transverse to $T_{i}^{2}$. Recall that under a single T-duality $R \rightarrow 1 / R$ the string coupling transforms as $e^{\phi} \rightarrow$ $e^{\phi} / R$. It follows that under six T-dualities, the four moduli $S, T_{i}$ go to the inverse gauge couplings of the corresponding $D 3$ and $D 7_{i}$ branes:

$\operatorname{Im} S \rightarrow \frac{1}{g_{3}^{2}}=e^{-\phi} \quad \operatorname{Im} T_{i} \rightarrow \frac{1}{g_{7 i}^{2}}=e^{-\phi} \mathcal{V} / v_{i}$

with $\mathcal{V}=v_{1} v_{2} v_{3}$ the total internal volume and $e^{\phi}$ is the 10dimensional string coupling $g_{s}=e^{\phi}$. The Kähler potential (10) then becomes

$\mathcal{K} \rightarrow-2 \ln \left(e^{-2 \phi} \mathcal{V}\right)=-\ln (S-\bar{S})-2 \ln \hat{\mathcal{V}}$

where $\hat{\mathcal{V}}=e^{-3 \phi / 2} \mathcal{V}$. 
Corrections to the Kähler potential in type II strings (with D-branes), are induced through corrections of the Einstein graviton kinetic terms. The perturbative corrections in $\alpha^{\prime}, \hat{\xi}$, and the string one-loop corrections $\hat{\delta}$, both arise in the string frame as corrections to the Einstein kinetic terms [34]:

$$
\left[e^{-2 \phi}(\mathcal{V}+\hat{\xi})+\hat{\delta}\right] \mathcal{R}
$$

where $\hat{\xi}$ is of order $\alpha^{\prime 3}$, arising at four loops in the CalabiYau $\sigma$-model, and is proportional to the Euler number of the Calabi-Yau manifold $\chi, \hat{\xi}=-\left[\zeta(3) / 4(2 \pi)^{3}\right] \chi[8,35,36]$, and $\hat{\delta}$ is in general a function of moduli fields.

From the above form, it follows that the two corrections can be accounted for by a shift of the Calabi-Yau volume $\mathcal{V}$ and of the inverse $4 \mathrm{~d}$ closed string coupling $e^{-2 \phi_{4}}=e^{-2 \phi} \mathcal{V}$ :

$\mathcal{V} \rightarrow \mathcal{V}+\xi ; \quad e^{-2 \phi_{4}}=e^{-2 \phi} \mathcal{V} \rightarrow e^{-2 \phi_{4}}+\hat{\delta}$

It is now clear that the radiatively corrected Kähler potential reads:

$$
\begin{aligned}
\mathcal{K} & =-2 \ln \left[e^{-2 \phi}(\mathcal{V}+\hat{\xi})+\hat{\delta}\right] \\
& =-\ln e^{-\phi}-2 \ln (\hat{\mathcal{V}}+\xi+\delta) \\
& =-\ln (S-\bar{S})-2 \ln (\hat{\mathcal{V}}+\xi+\delta),
\end{aligned}
$$

where $\hat{\mathcal{V}}$ is defined in (13) and

$$
\xi=\hat{\xi} / g_{s}^{3 / 2}=-\frac{\zeta(3)}{4(2 \pi)^{3} g_{s}^{3 / 2}} \chi ; \quad \delta=\hat{\delta} g_{s}^{1 / 2} .
$$

Note that a nonvanishing $\xi$ in the large volume limit gives rise to localised graviton kinetic terms in the internal CalabiYau space at the points where the Euler number is concentrated. Indeed it remains finite in the large volume limit in (14) leading to a localised Einstein action, on an effective 3-brane, studied in [34].

These localised graviton kinetic terms can generate one loop corrections that grow logarithmically with the size of the bulk in the presence of 7-brane sources [27]. Indeed, the localised graviton vertices can emit closed strings propagating along all the six dimensions of the internal space. The contribution of the relevant diagrams contains the exchange of these closed strings in the bulk between a certain number of graviton vertices from the $4 \mathrm{~d}$ Einstein action localised in the internal space and another boundary that can be a $D$-brane or an orientifold plane. These diagrams correspond to local tadpoles whose existence can be consistent with global tadpole cancellation. Each of branes/orientifold planes behave as point-like sources in the corresponding transverse space. The emitted closed string from the localised graviton vertices carry in principle momentum along all the six internal dimensions. However, the momentum along the directions parallel to the worldvolume of brane/orientifold plane vanishes by conservation. It follows that the exchanged closed strings carry only transverse momentum $p_{\perp}$ which is not conserved due to the presence of branes/orientifold planes that break translation invariance in the transverse directions. Thus the relevant diagram that contributes to $\delta$, in the large transverse volume $V_{\perp}$ limit, takes the form:

$\delta \sim \frac{1}{V_{\perp}} \sum_{\left|p_{\perp}\right|<M_{s}} \frac{1}{p_{\perp}^{2}} F\left(\vec{p}_{\perp}\right) ; \quad \vec{p}_{\perp}=\left(\frac{n_{1}}{R}, \ldots, \frac{n_{d}}{R}\right)$,

where $F\left(\vec{p}_{\perp}\right)$ are the local tadpoles in the momentum space and the summation (instead of integration) is because $\vec{p}_{\perp}$ are discrete in the compact transverse space that we parameterise its size as $V_{\perp} \sim R^{d}$. The tadpoles arise from the distribution of D-branes and orientifolds which act as classical point-like sources in the transverse space. Considering for instance $2^{d}$ orientifolds located at the corners of a $d$-dimensional cube formed by $d$ dimensions of equal size $\pi R$ and a brane at the position $\vec{y}$ (plus its images), the local tadpole is given by:

$F\left(\vec{p}_{\perp}\right) \sim\left\{\prod_{i=1}^{d}\left(\frac{1+(-)^{n_{i}}}{2}\right)-\cos \left(\vec{p}_{\perp} \vec{y}\right)\right\}$.

It follows that its contribution to the amplitude (18) would contain an infrared divergence in the large transverse limit when its co-dimension is less or equal to 2 . The divergence is linear in $R$ for $d=1$ and logarithmic for $d=2$, while the amplitude is finite for $d>2$.

In conclusion, in the system with 7-branes and localised graviton kinetic terms in the internal space, the effective twodimensional propagation of closed strings induce an infrared divergence in the loop correction that goes logarithmically when the co-dimension 2 transverse dimension is large [27]. Due to the infrared divergence, one could also expect it is the dominant correction at that order in the string loop expansion. One could thus write Eq. (18) as

$\delta=\eta \ln u$,

where $\eta$ is some model dependent constant and $u$ is the modulus of the space transverse to a D7-brane.

We would like to emphasise again that the necessary condition for the arguments of [27] is to have localised kinetic terms in the internal space. Here we have to discuss separately the case of smooth Calabi-Yau manifolds and orbifolds. As argued before, the presence of a non-vanishing $\xi$ at the string tree-level can induce at one loop level logarithmic corrections of the type Eq. (20). An explicit computation however is rather difficult to be performed since it requires quantising strings propagating in Calabi-Yau threefolds taking into account the perturbative in $\alpha^{\prime}$ correction (thus treating it exactly), and it is not within the scope of the present work.

In orbifold compactifications of type IIB orientifolds, the $\alpha^{\prime}$ correction $\xi$ vanishes. Thus at the leading order in string 
loop expansion, graviton kinetic terms in Eq. (14) are tendimensional and therefore the arguments of [27] do not apply. However this is not the case at higher orders. The one loop correction $\hat{\delta}$ receives moduli dependent contributions only from $N=2$ supersymmetric sectors depending on the moduli of the corresponding fixed torus under the action of the orbifold group [37]. For D7-branes transverse to the 2-torus $T^{2}$, it is given by a sum over BPS states corresponding to the open string winding modes where $N=2$ vector multiplets and hypermultiplets contribute with opposite signs. The result depends on the complex structure modulus of the torus but not on its volume and does not contain any logarithmic correction, as expected from our general analysis above. Thus, one loop corrections in the Kähler potential cannot lead to logarithmic dependence, in agreement with one-loop results in the literature for orbifolds (see for instance [10,37]). On the contrary, the kinetic function of $D 7$ gauge fields which are localised in the transverse dimension receive large corrections that grow logarithmically with the transverse volume, which is calculated explicitly in [37]. Notice however, that the one loop corrections [10] contain terms localised on the transverse $T^{2}$ and thus two loop corrections are expected to diverge logarithmically with its volume, following the argument above. In this case, the correction $\delta$ in Eqs. (17) and (20) should have an additional factor of $g_{s}^{2} \ln u$.

In the following, we will consider a radiatively corrected Kähler potential (16) with Eq. (20) $\delta=\eta \ln u$, :

$\mathcal{K}=-\ln (S-\bar{S})-2 \ln (\hat{\mathcal{V}}+\xi+\eta \ln u)$.

\subsection{D-terms in the presence of $D 7$-branes}

It has been suggested that magnetised branes along $(1,1)$ cycles of the internal compactification space can be used to stabilise the Kähler moduli, as an alternative to nonpertubative effects, at a de Sitter vacuum through the induced D-terms [20-22]. The advantage of magnetic fluxes on $D$ branes, as opposed to non-perturbative effects and $\overline{D 3}$ contributions, is that these have an exact string description at weak coupling (i.e. to all orders in $\alpha^{\prime}$ ) and can be studied within the standard effective supergravity. In this subsection we will discuss the D-term contributions from magnetised $D 7$-branes in type IIB superstring theory. We will assume that all complex structure moduli and the axiondilaton ten-dimensional (10d) field are fixed in a standard way by appropriate 3 -form fluxes at a vacuum preserving $N=1$ supersymmetry in four dimensions with weak string coupling. Moreover, we shall consider zero vacuum expectation values (VEVs) for all charged fields and restrict our analysis to the Kähler moduli associated with the world- and transverse-volumes of the D7-branes; they should all be considered large in string units for the consistency of the effective supergravity description.
In usual D7-brane configurations representing supersymmetric four-dimensional (4d) effective theories there are stacks of branes associated with some non-abelian gauge group while it is common that additional branes intersect each other. A single D7-brane spans four compact dimensions and forms a two-cycle intersection with any other nonoverlapping brane.

We now consider a IIB/F-theory framework with the presence of intersecting 7-branes. Stacks of D7-branes are associated with gauge groups and we assume a $D$-brane configuration where some anomalous $U(1)$ is present, induced by a corresponding magnetic flux. A 4-cycle Kähler modulus $T_{a}$ associated with the world-volume of the magnetised $D 7$ brane acquires then a charge $Q$ under the $U(1)$ as a shift symmetry along its real component: $T^{a} \rightarrow T^{a}+Q \omega$, with $\omega$ the transformation parameter (the appropriate topological conditions for this, are discussed for instance in [24]). In general, we also expect the existence of complex scalar fields $\phi^{J}$ carrying charges $Q_{J}$.

The induced D-term has the generic form dictated by the effective $N=1$ supergravity [18,19,23,24]:

$V_{D}=\frac{g_{D 7}^{2}}{2}\left(i Q \partial_{T^{a}} \mathcal{K}\left(T^{a}\right)+\sum_{J} Q_{J}\left|\left\langle\phi^{J}\right\rangle\right|^{2}\right)^{2}$

where the gauge coupling is fixed by the kinetic function: $\frac{1}{g_{D 7}^{2}}=\operatorname{Im}\left(T^{a}\right)$ and $\phi^{J}$ are scalar components of superfields whose charges $Q_{J}$ are subject to anomaly cancellation conditions (that are automatically satisfied in a consistent string background) [21]. Although in general the VEVs of the scalar fields are on-zero, for our present purposes we can ignore the matter fields and write (22) as follows

$V_{D}=-\frac{d_{a}}{2 \operatorname{Im}\left(T^{a}\right)}\left(\partial_{T^{a}} \mathcal{K}\left(T^{a}\right)\right)^{2}$,

in which $d_{a}=Q^{2}$.

In our convention, we denote the imaginary part of the world-volume Kähler modulus $T_{a}$ as $\tau_{a}$. The whole 6dimensional volume can be expressed as sum of triple products of 2-cycle moduli:

$\mathcal{V}=\frac{1}{6} \kappa_{a b c} v^{a} v^{b} v^{c}$

where $\kappa_{a b c}$ are the triple intersection numbers. In the framework of 3 intersecting $D 7$-branes, we take 2-cycle $v^{a}$ as the transverse volume modulus of each $D 7$-brane with worldvolume $\tau_{a}$ :

$v_{a}=\frac{\mathcal{V}}{\tau_{a}}$,

\footnotetext{
${ }^{2}$ See also argument in [23].
} 
and take $\kappa_{a b c}$ as $\epsilon_{a b c}$ for simplicity. Then the volume can be expressed as

$\mathcal{V}=v_{1} v_{2} v_{3}=\sqrt{\tau_{1} \tau_{2} \tau_{3}}$

\section{Volume stabilisation from intersecting $D 7-B$ ranes}

In this section we investigate the implications of $D 7$-branes on the stabilisation of Kähler moduli. Starting with the simplest case, we introduce only one $D 7$-brane and observe that this is not adequate to stabilise all moduli. Then, we proceed with the inclusion of three intersecting $D 7$-branes.

\subsection{A single $D 7$-brane}

We start with a single space-time filling $D 7$-brane and assume that all the complex structure moduli and the axiondilaton are stabilised by fluxes. The Kähler modulus can be divided into the world volume part of the D7-brane $\tau$ and the transverse part $u$. Both $\tau$ and $u$ are real 4-cycle volumes. Then we can write the compactifacation volume $\mathcal{V}$ in terms of the two Kähler moduli as follows:

$\mathcal{V}=\tau \sqrt{u}$.

The no-scale structure is broken by perturbative corrections: $\alpha^{\prime}$ world-sheet corrections and string loop corrections. The Kähler potential now takes the general form:

$\mathcal{K}=-2 \ln (\tau \sqrt{u}+\xi+\eta \ln (u))$,

The string loop correction term $\eta \ln (u)$ is of course valid in the perturbative region:

$|\eta \ln (u)|<\tau \sqrt{u}$

The corresponding F-term potential with a superpotential $\mathcal{W}_{0}$ is:

$V_{F}=\frac{\mathcal{W}_{0}^{2}(-8 \eta+3 \xi+3 \eta \ln (u))}{(8 \eta+2 \tau \sqrt{u}-\xi-\eta \ln (u))(\tau \sqrt{u}+\xi+\eta \ln (u))^{2}}$.

In the large volume expansion, we can compute the derivative with respect to $u$ :

$\frac{d V_{F}(\tau, u)}{d u}=-\eta \mathcal{W}_{0}^{2} \frac{3(-10+3 \ln (u))}{4 \tau^{3} u^{5 / 2}}+O\left(\eta^{2}\right)+O(\xi)$.

We find that for $\eta$ being negative, the potential has a minimum in the $u$ direction. Thus, the string loop correction $\eta \ln (u)$ can stabilise the transverse direction of the D7-brane. However, for the volume part $\tau$, the first derivative doesn't show the stabilisation. Indeed, in the appendix we show that even in the presence of an uplifting D-term, there is no $\mathrm{dS}$ minimum with just perturbative corrections for a single $D 7$-brane.
3.2 Stabilisation of the total volume by three intersecting D7-branes

In the same way, we can get the F-term potential for 2 nonparallel D7-branes and find that there is always one Kähler modulus which is not stabilised. Thus, in order to stabilise all the Kähler moduli, we should consider that there exist at least three non-parallel (magnetised) D7-branes. This corresponds to 3 intersecting D7-branes which is quite general in string model building. In the following, we neglect the $\alpha^{\prime}$ correction and consider only the string loop correction. The general Kähler potential can be written as:

$$
\begin{aligned}
\mathcal{K} & =-2 \ln \left(\sqrt{\tau_{1} \tau_{2} \tau_{3}}+\sum_{i} 2 \eta_{i} \ln \left(\frac{\mathcal{V}}{\tau_{i}}\right)\right) \\
& =-2 \ln \left(\sqrt{\tau_{1} \tau_{2} \tau_{3}}+\sum_{i} \eta_{i}^{\prime} \ln \left(\tau_{i}\right)\right), \quad \eta_{a}^{\prime}=\sum_{i} \eta_{i}-2 \eta_{a} .
\end{aligned}
$$

Each $\tau_{i}$ corresponds to the world volume of one D7-brane real 4-cycle. We calculate the first derivative with respect to either $\tau_{a}$ :

$$
\begin{aligned}
& \frac{d V_{F}\left(\tau_{1}, \tau_{2}, \tau_{3}\right)}{d \tau_{a}} \\
& =\mathcal{W}_{0}^{2} \frac{3\left(\sum_{i \neq a}\left(8 \eta_{i}^{\prime}-3 \eta_{i}^{\prime} \ln \left(\tau_{i}\right)\right)+10 \eta_{a}^{\prime}-3 \eta_{a}^{\prime} \ln \left(\tau_{a}\right)\right)}{4 \prod_{i \neq a} \tau_{i}^{\frac{3}{2}} \tau_{a}^{\frac{5}{2}}} \\
& \quad+O\left(\eta^{\prime 2}\right) .
\end{aligned}
$$

The minimisation condition from the three directions in Eq. (34) then shows that a minimum only exists for the total volume $\mathcal{V}$ if

$\eta_{1}=\eta_{2}=\eta_{3}=\eta_{\tau}<0$

The other two directions, which can be thought of as the ratios between $\tau_{1}, \tau_{2}$ and $\tau_{3}$, remain flat, since under the condition (35), the Kähler potential and the corresponding Fterm potential only depend on $\mathcal{V}$. Indeed, the Kähler potential is:

$\mathcal{K}=-2 \ln \left(\mathcal{V}+2 \eta_{\tau} \ln (\mathcal{V})\right)$,

and the F-part of the effective potential is

$$
\begin{aligned}
V_{F}(\mathcal{V}) & =-\frac{3 \eta_{\tau} \mathcal{W}_{0}^{2}\left(2 \eta_{\tau}+4 \mathcal{V}+4 \eta_{\tau} \ln (\mathcal{V})-\mathcal{V} \ln (\mathcal{V})\right)}{\left(\mathcal{V}+2 \eta_{\tau} \ln (\mathcal{V})\right)^{2}\left(6 \eta_{\tau}^{2}+8 \eta_{\tau} \mathcal{V}+\mathcal{V}^{2}+\eta_{\tau}\left(4 \eta_{\tau}-\mathcal{V}\right) \ln (\mathcal{V})\right)} \\
& =\frac{\eta_{\tau} \mathcal{W}_{0}^{2}}{\mathcal{V}^{3}}(3 \ln (\mathcal{V})-12)+O\left(\eta_{\tau}^{2}\right)
\end{aligned}
$$

The first derivative of Eq. (37) shows that the minimum is independent of the $\eta_{\tau}$ parameter in the large volume limit:

$\frac{d V_{F}(\mathcal{V})}{d \mathcal{V}}=-\eta_{\tau} \frac{3 \mathcal{W}_{0}^{2}(3 \ln (\mathcal{V})-13)}{\mathcal{V}^{4}}+O\left(\eta_{\tau}^{2}\right)$ 


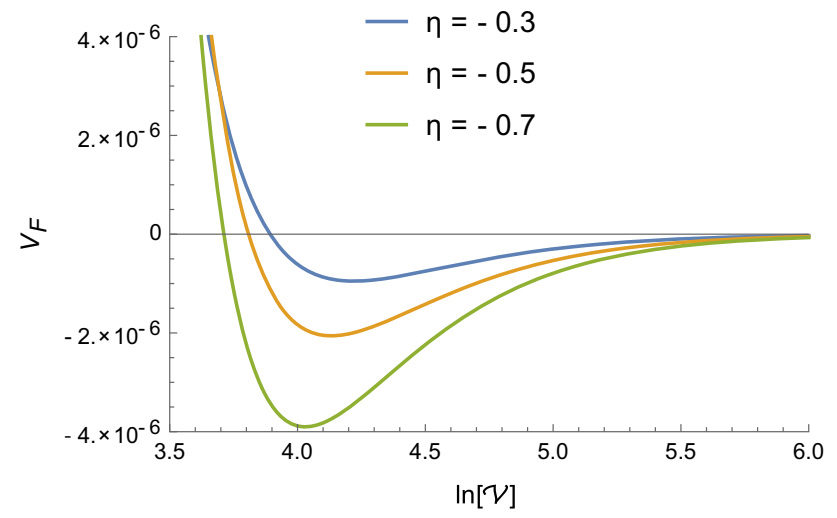

Fig. 1 The scalar potential of Eq. (37) for the choice $\mathcal{W}_{0}=1$

The potential (37) is plotted in Fig. 1.

At this minimum, the supersymmetric condition is not satisfied:

$D \mathcal{V} \mathcal{W}_{0}\left|\mathcal{V}_{\text {min }}=\partial \mathcal{V} K\right| \mathcal{V}_{\text {min }} \mathcal{W}_{0}=-\frac{2 \mathcal{W}_{0}}{\mathcal{V}_{\text {min }}}+O\left(\eta_{\tau}\right) \neq 0$,

so supersymmetry is spontaneously broken. The minimum is not stable due to the two undetermined directions and a tiny deviation from condition (35) would destabilise the total volume. We will discuss the stabilisation of the ratios in the next section where the condition (35) is not necessary.

There is a similar form to Eq. (36) in the classical large volume scenario. The equation of motion of the small cycle $\tau_{s}$ from Eq. (9) leads to

$\tau_{s} \propto \ln (\mathcal{V}) \propto \ln \left(\tau_{b}\right)$.

\subsection{D-term uplifting and ratios stabilisation}

In order to stabilise the ratios and find a dS vacuum, we introduce D-terms emerging from a magnetic flux on each D7-brane. These depend on the corresponding world volume modulus $\tau_{a}$ :

$$
\begin{aligned}
V_{D_{a}} & =\frac{d_{a}}{\tau_{a}}\left(\frac{\partial K}{\partial \tau_{a}}\right)^{2}=d_{a} \frac{\left(\mathcal{V}+2 \eta_{a}^{\prime}\right)^{2}}{\tau_{a}^{3}\left(\mathcal{V}+\sum_{i} \eta_{i}^{\prime} \ln \left(\tau_{i}\right)\right)^{2}} \\
& =\frac{d_{a}}{\tau_{a}^{3}}+O\left(\eta_{i}\right) .
\end{aligned}
$$

For simplicity, we still use the condition (35) to calculate the minimum. Notice that it is not a necessary condition once Dterms are included from all three $D 7$-brane stacks, leading to a global minimum for all the Kähler moduli.

We choose $\tau_{1}, \tau_{2}$ and $\mathcal{V}$ as the 3 independent dynamical variables. The sum of the F-term potential (38) and D-term potentials (43) in the large volume limit becomes:

$$
V_{\text {sum }}=\frac{\eta_{\tau} \mathcal{W}_{0}^{2}}{\mathcal{V}^{3}}(3 \ln (\mathcal{V})-12)+\frac{d_{1}}{\tau_{1}^{3}}+\frac{d_{2}}{\tau_{2}^{3}}+\frac{d_{3} \tau_{1}^{3} \tau_{2}^{3}}{\mathcal{V}^{6}}
$$

The minimisation conditions of $\tau_{1}$ and $\tau_{2}$ lead directly to

$$
\begin{aligned}
\tau_{1}^{3} & =\left(\frac{d_{1}^{2}}{d_{2} d_{3}}\right)^{\frac{1}{3}} \mathcal{V}^{2} \\
\tau_{2}^{3} & =\left(\frac{d_{2}^{2}}{d_{1} d_{3}}\right)^{\frac{1}{3}} \mathcal{V}^{2} .
\end{aligned}
$$

Substituting these expresssions into the minimisation condition of $\mathcal{V}$ we get:

$\eta_{\tau} \mathcal{W}_{0}^{2}(13-3 \ln (\mathcal{V}))=2\left(d_{1} d_{2} d_{3}\right)^{\frac{1}{3}} \mathcal{V}$

There are two conditions that must be satisfied in order to get a dS minimum.

- The first is that there should exist two real solutions of Eq. (46) where the smaller one corresponds to a minimum and the larger one corresponds to a maximum. Indeed, by doing a change of variables in Eq. (46), we get:

$z e^{z}=\frac{2 e^{\frac{13}{3}}\left(d_{1} d_{2} d_{3}\right)^{\frac{1}{3}}}{3 \eta_{\tau} \mathcal{W}_{0}^{2}} ; z=\frac{13}{3}-\ln (\mathcal{V})$

The first equation above has two solutions for $z$ negative while the function $z e^{z}$ has a minimum at $z=-1$. Thus, the right hand side should be between $-e^{-1}$ and 0 that requires:

$$
-\frac{3 e^{-\frac{16}{3}}}{2} \simeq-0.007242<\frac{\left(d_{1} d_{2} d_{3}\right)^{\frac{1}{3}}}{\eta_{\tau} \mathcal{W}_{0}^{2}}<0 .
$$

The smaller solution $\mathcal{V}_{0}$ of Eq. (46) is:

$\mathcal{V}_{0}=e^{\frac{13}{3}-W\left[\frac{2 e^{\frac{13}{3}}\left(d_{1} d_{2} d_{3}\right)^{\frac{1}{3}}}{3 \eta_{\tau} \mathcal{W}_{0}^{2}}\right]}$

in which $\mathrm{W}$ is the Lambert W-Function.

- The second condition is that the potential should be positive at the minimum. Using Eqs. (45) and (46), we can express the potential at the minimum in a simple form:

$$
V_{\text {sum }}^{\min }=\frac{\eta_{\tau} \mathcal{W}_{0}^{2}}{\mathcal{V}_{0}^{3}}+\frac{\left(d_{1} d_{2} d_{3}\right)^{\frac{1}{3}}}{\mathcal{V}_{0}^{2}}>0
$$

Solving it numerically gives a new constraint

$$
\frac{\left(d_{1} d_{2} d_{3}\right)^{\frac{1}{3}}}{\eta_{\tau} \mathcal{W}_{0}^{2}}<-0.006738
$$




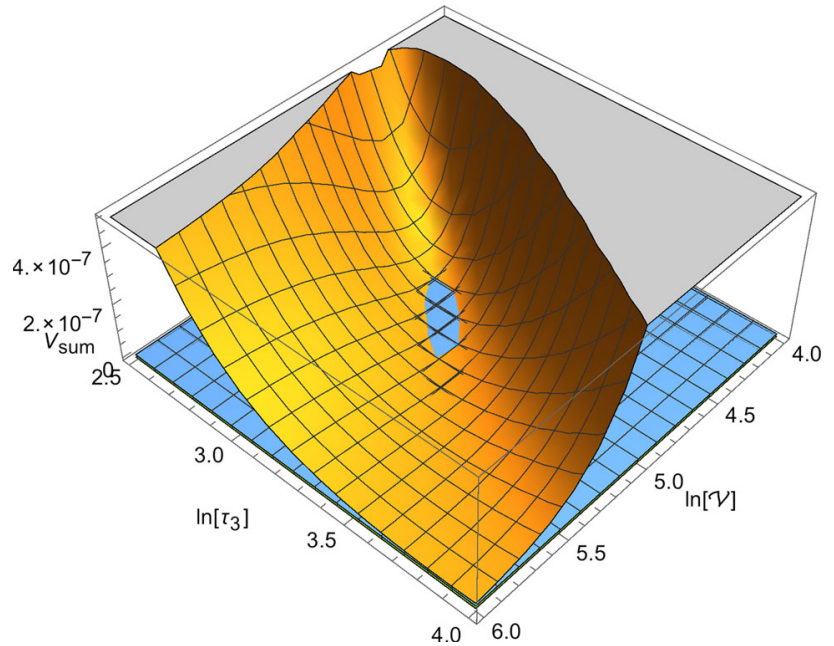

Fig. 2 Sum of the F- and D-term contributions to the potential (37) and (42) in terms of $\ln (\mathcal{V})$ and $\ln \left(\tau_{3}\right)$ with the choice $\mathcal{W}_{0}=1, \eta_{\tau}=$ $-0.4, d_{1}=d_{2}=0.00375, d_{3}=0.0018$. The blue area corresponds to $V=10^{-8}$ plane

which is consistent with the inequalities (48) and together lead to

$$
-0.007242<\frac{\left(d_{1} d_{2} d_{3}\right)^{\frac{1}{3}}}{\eta_{\tau} \mathcal{W}_{0}^{2}}<-0.006738
$$

Within this range, we can get approximately the order of magnitude of the volume $\mathcal{V}_{0}$ :

$\ln \left(\mathcal{V}_{0}\right) \simeq 5$

which corresponds to a Grand Unification Theory (GUT) scale compactification volume.

We show an example in Fig. 2, in which we take $d_{1}=d_{2}$, thus $\tau_{1}=\tau_{2}$ at minimum according to Eq. (45).

\section{Conclusions}

Moduli stabilisation in string theory is a long standing issue and despite the significant progress that has been made during the last two decades, the proposed solutions are still far from being conclusive. The main ingredients of the existing scenarios are backgound fluxes, string loop-corrections and non-perturbative effects.

The key point towards a convincing solution is to implement a realistic dynamical mechanism which generates a scalar potential and provides masses to the various massless scalar fields emerging in string compactifications. In this work, we have studied this problem in the framework of IIB/F-theory compactifications and we have proposed a new geometric mechanism which dispences with the use of non-perturbative effects. We have considered configurations, where the main ingedients are intersecting $D 7$-branes equipped with internal magnetic fluxes which have an exact description to all orders in $\alpha^{\prime}$, and we have investigated their implications on the stabilisation of the Kähler moduli. More concretely, assuming that the VEVs of the complex structure moduli and of the axion-dilaton field are already fixed by supersymmetry conditions, we examined the modifications of the Kähler potential arising from perturbative $\alpha^{\prime}$ and loop corrections. Elaborating on the essential features of $D 7$ branes in the configuration of the compact space, we concluded that in the transverse large volume limit of dimension two, the effective action receives loop corrections which are logarithmically divergent. In effect, the Calabi-Yau volume in the Kähler potential receives corrections which display logarithmic dependence on the size of the transverse to the $D 7$-branes directions. This is in contrast to the $\alpha^{\prime}$ correction which induces just a shift to the volume by a constant parameter $\xi$.

In addition, magnetised $D 7$-branes, have significant implications on the stabilisation of the Kähler moduli and, at the same time, they can naturally ensure the existence of a dS minimum. More precisely, magentised $D 7$-branes are associated with anomalous $U(1)$ symmetries which are also a source of D-terms, that depend on the world-volume of the corresponding $D 7$-branes. These contributions to the effective potential can stabilise the ratios between each worldvolume modulus and the total volume and, thus, they work as an uplift mechanism to realise de-Sitter minima. To show this we have computed the scalar potential and performed a detailed analysis, where we found that in the case of one D7-brane the logarithmic shift of the volume stabilises only the modulus of the transverse space. Stabilisation of the total volume is achieved only in the presence of at least three intersecting $D 7$-branes, which span all six dimensions of the compact space. Interestingly, in this scenario, non-perturbative corrections are not necessary.

The realisation of this geometric stabilisation mechanism and the uplifting is a viable scenario in F-theory [38] where intersecting 7-branes are a natural phenomenon which also has additional attractive features beyond the present context. For instance, one could use the F-theory model building [39] to realise the Standard Model.

Acknowledgements This work was supported in part by the Swiss National Science Foundation, in part by Labex "Institut Lagrange de Paris" and in part by a CNRS PICS grant. G.K.L. would like to thank the LPTHE in Paris and the ITP in Bern for their kind hospitality while Y.C. would like to thank ITP, where part of the work was completed. Y.C. also thanks Karim Benakli, Emilian Dudas and Mark D. Goodsell for discussions.

Open Access This article is distributed under the terms of the Creative Commons Attribution 4.0 International License (http://creativecomm 
ons.org/licenses/by/4.0/), which permits unrestricted use, distribution, and reproduction in any medium, provided you give appropriate credit to the original author(s) and the source, provide a link to the Creative Commons license, and indicate if changes were made. Funded by SCOAP ${ }^{3}$.

\section{Appendix}

The D-term potential from a $U(1)$ magnetic flux on a single D7-brane has the form:

$$
V_{D}=d \frac{u}{\tau(\tau \sqrt{u}+\xi+\eta \ln (u))^{2}} .
$$

The corresponding scalar potential is:

$$
\begin{aligned}
V= & \frac{\mathcal{W}_{0}^{2}(-8 \eta+3 \xi+3 \eta \ln (u))}{(8 \eta+2 \tau \sqrt{u}-\xi-\eta \ln (u))(\tau \sqrt{u}+\xi+\eta \ln (u))^{2}} \\
& +d \frac{u}{\tau(\tau \sqrt{u}+\xi+\eta \ln (u))^{2}} .
\end{aligned}
$$

In order that the scalar potential has a dS minimum, one necessary condition is that for the direction along $\mathcal{V}=\tau \sqrt{u}$, there exists a dS minimum when $u$ is a constant. It is the same for the direction along $\tau$ since they only differ by a factor of $\sqrt{u}$ which is a positive constant. We can thus write the potential in terms of $\mathcal{V}$ and take the other parameters including $u$ as constants:

$$
\begin{aligned}
V(\mathcal{V}) & =\frac{a}{(\mathcal{V}-b)(\mathcal{V}-c)^{2}}+\frac{e}{\mathcal{V}(\mathcal{V}-c)^{2}} \\
& =\frac{(a+e) \mathcal{V}-b e}{\mathcal{V}(\mathcal{V}-b)(\mathcal{V}-c)^{2}}
\end{aligned}
$$

The potential should be positive when $\mathcal{V} \rightarrow+\infty$. So $(a+$ $e$ ) should be positive. Since we only consider the existence of a dS minimum, independently of the overall normalisation of the potential, we can divide it by $(a+e)$ and define a new parameter $f=\frac{b e}{a+e}$. The potential now becomes:

$\frac{V(\mathcal{V})}{a+e}=\frac{\mathcal{V}-f}{\mathcal{V}(\mathcal{V}-b)(\mathcal{V}-c)^{2}}$

and has three singularities at $0, b$ and $c$. The dS minimum should lie in the branch outside these three singularities. Thus, we define a new parameter

$g=\max (0, b, c)$,

and only consider the region:

$\mathcal{V}>g$.

First, consider the case that $f>g$; we find that the potential becomes negative in the range $g<\mathcal{V}<f$, which means there is no dS minimum. We turn to the case $f \leq g$ and calculate the first derivative with respect to $\mathcal{V}$ :

$$
\begin{aligned}
& \frac{d V(\mathcal{V})}{d \mathcal{V}} /(a+e) \\
& =\frac{1}{\mathcal{V}(\mathcal{V}-b)(\mathcal{V}-c)^{2}}-\frac{\mathcal{V}-f}{\mathcal{V}^{2}(\mathcal{V}-b)(\mathcal{V}-c)^{2}} \\
& \quad-\frac{\mathcal{V}-f}{\mathcal{V}(\mathcal{V}-b)^{2}(\mathcal{V}-c)^{2}}-\frac{2(\mathcal{V}-f)}{\mathcal{V}(\mathcal{V}-b)(\mathcal{V}-c)^{3}}
\end{aligned}
$$

Suppose $b$ is the largest singularity $b=g$. The first derivative becomes:

$$
\begin{aligned}
\frac{d V(\mathcal{V})}{d \mathcal{V}} /(a+e)= & \frac{f-b}{\mathcal{V}(\mathcal{V}-b)^{2}(\mathcal{V}-c)^{2}} \\
& -\frac{\mathcal{V}-f}{\mathcal{V}^{2}(\mathcal{V}-b)(\mathcal{V}-c)^{2}} \\
& -\frac{2(\mathcal{V}-f)}{\mathcal{V}(\mathcal{V}-b)(\mathcal{V}-c)^{3}}
\end{aligned}
$$

Note that all the terms above are negative in the region $f \leq$ $g<\mathcal{V}$. Thus, there is no minimum in this region. The same results hold for 0 or $c$ to be the largest singularity $g$. Thus, no $\mathrm{dS}$ minimum exists in the physical region of the parameter space.

\section{References}

1. A.R. Frey, J. Polchinski, Phys. Rev. D 65, 126009 (2002). arXiv:hep-th/0201029

2. S. Kachru, M.B. Schulz, S. Trivedi, JHEP 0310, 007 (2003) arXiv:hep-th/0201028

3. K. Dasgupta, G. Rajesh, S. Sethi, JHEP 9908, 023 (1999). arXiv:hep-th/9908088

4. T.R. Taylor, C. Vafa, Phys. Lett. B 474, 130 (2000). arXiv:hep-th/9912152

5. S. Kachru, R. Kallosh, A.D. Linde, S.P. Trivedi, Phys. Rev. D 68 , 046005 (2003). arXiv:hep-th/0301240

6. J.P. Derendinger, L.E. Ibanez, H.P. Nilles, Phys. Lett. B 155, 65 (1985)

7. J.P. Derendinger, L.E. Ibanez, H.P. Nilles, Nucl. Phys. B 267, 365 (1986)

8. K. Becker, M. Becker, M. Haack, J. Louis, JHEP 0206, 060 (2002). arXiv:hep-th/0204254

9. G. von Gersdorff, A. Hebecker, Phys. Lett. B 624, 270 (2005). arXiv:hep-th/0507131

10. M. Berg, M. Haack, B. Körs, JHEP 0511, 030 (2005). arXiv:hep-th/0508043

11. M. Berg, M. Haack, B. Körs, Phys. Rev. Lett. 96, 021601 (2006). arXiv:hep-th/0508171

12. S.L. Parameswaran, A. Westphal, JHEP 0610, 079 (2006). arXiv:hep-th/0602253

13. M. Cicoli, J.P. Conlon, F. Quevedo, JHEP 0801, 052 (2008). arXiv:0708.1873 [hep-th]

14. M. Berg, M. Haack, J.U. Kang, S. Sjörs, JHEP 1412, 077 (2014). arXiv:1407.0027 [hep-th]

15. M. Haack, J.U. Kang, JHEP 1602, 160 (2016). arXiv:1511.03957 [hep-th]

16. T. Kobayashi, N. Omoto, H. Otsuka, T .H. Tatsuishi, Phys. Rev. D 97(10), 106006 (2018). arXiv:1711.10274 [hep-th]

17. M. Haack , J. U. Kang. arXiv: 1805.00817 [hep-th]

18. C.P. Burgess, R. Kallosh, F. Quevedo, JHEP 0310, 056 (2003). arXiv:hep-th/0309187 
19. H. Jockers, J. Louis, Nucl. Phys. B 705, 167 (2005). arXiv:hep-th/0409098

20. I. Antoniadis, T. Maillard, Nucl. Phys. B 716, 3 (2005). arXiv:hep-th/0412008

21. I. Antoniadis, A. Kumar, T. Maillard, hep-th/0505260 and Nucl. Phys. B 767, 139 (2007). arXiv:hep-th/0610246

22. I. Antoniadis, J.-P. Derendinger, T. Maillard, Nucl. Phys. B 808, 53 (2009). arXiv:0804.1738 [hep-th]

23. A. Achucarro, B. de Carlos, J.A. Casas, L. Doplicher, JHEP 0606, 014 (2006). arXiv:hep-th/0601190

24. M. Haack, D. Krefl, D. Lüst, A. Van Proeyen, M. Zagermann, JHEP 0701, 078 (2007). arXiv:hep-th/0609211

25. V. Balasubramanian, P. Berglund, J.P. Conlon, F. Quevedo, JHEP 0503, 007 (2005). arXiv:hep-th/0502058

26. J.P. Conlon, F. Quevedo, K. Suruliz, JHEP 0508, 007 (2005). arXiv:hep-th/0505076

27. I. Antoniadis, C. Bachas, Phys. Lett. B 450, 83 (1999). arXiv:hep-th/9812093

28. I. Antoniadis, K. Benakli, A. Laugier, T. Maillard, Nucl. Phys. B 662, 40 (2003). arXiv:hep-ph/0211409

29. S. Gukov, C. Vafa, E. Witten, Nucl. Phys. B 584 (2000) 69 Erratum: [Nucl. Phys. B 608 (2001) 477] arXiv:hep-th/9906070
30. P. Candelas, X. de la Ossa, Nucl. Phys. B 355, 455 (1991)

31. S.B. Giddings, S. Kachru, J. Polchinski, Phys. Rev. D 66, 106006 (2002). arXiv:hep-th/0105097

32. D. Lüst, P. Mayr, S. Reffert, S. Stieberger, Nucl. Phys. B 732, 243 (2006). arXiv:hep-th/0501139

33. Y. Honma, H. Otsuka, Phys. Lett. B 774, 225 (2017). arXiv:1706.09417 [hep-th]

34. I. Antoniadis, R. Minasian, P. Vanhove, Nucl. Phys. B 648, 69 (2003). arXiv:hep-th/0209030

35. M.T. Grisaru, A.E.M. van de Ven, D. Zanon, Nucl. Phys. B 277, 409 (1986)

36. I. Antoniadis, S. Ferrara, R. Minasian, K.S. Narain, Nucl. Phys. B 507, 571 (1997). arXiv:hep-th/9707013

37. I. Antoniadis, C. Bachas, C. Fabre, H. Partouche, T.R. Taylor, Nucl. Phys. B 489, 160 (1997). arXiv:hep-th/9608012

38. C. Vafa, Nucl. Phys. B 469, 403 (1996). arXiv:hep-th/9602022

39. C. Beasley, J.J. Heckman, C. Vafa, JHEP 0901, 058 (2009). arXiv:0802.3391 [hep-th] 